\title{
Reconfigurable Cooperative Control of Networked Lagrangian Systems Under Actuator Saturation Constraints
}

\author{
A. R. Mehrabian, S. Tafazoli, and K. Khorasani
}

\begin{abstract}
In this paper, a reconfigurable control strategy is proposed for state synchronization and tracking control of networked (electro-) mechanical Euler-Lagrange (EL) systems that are subject to input saturation constraints that may arise due to actuator faults or failures. The reconfigurable controller consists of three main parts. The first part, known as the nominal controller, is a distributed controller that is employed to guarantee global stability of the multiagent networked EL system provided that certain mild connectivity conditions are satisfied in absence or presence of actuator saturation constraints. The second part, known as the reconfigured controller, is a constrained nonlinear smooth distributed controller that has a different structure and gains from the nominal controller. This controller can preserve the overall control objectives in presence of actuator faults and actuator saturation constraints. The third part is a switching strategy between the nominal and the reconfigured controllers. Global stability as well as asymptotic convergence of the synchronization and the tracking errors to origin for switchings under certain conditions between the nominal and the reconfigured controllers with non-vanishing dwell-times for a fixed network topology are shown to be guaranteed. Simulation results are reported to demonstrate and validate the merits of the proposed controllers.
\end{abstract}

\section{INTRODUCTION}

Cooperative control in networked systems is to be realized by information exchanges among agents. This behavior and property is defined as the ability to synchronize the agents' states (or outputs) in order to accomplish complex tasks that otherwise could not have been accomplished by only utilizing individually decoupled and non-interacting agents. Cooperative control of networked (electro-) mechanical Euler-Lagrange (EL) systems have applications in various fields. These applications include cooperative control of multiple uninhabited (or unmanned) aerial vehicles (UAVs) and uninhabited ground vehicles (UGVs) for search and rescue operations; intelligence, surveillance, and reconnaissance (ISR) missions; and mapping unknown or partially known environments, among others. It has also

This research is supported in part by a grant from the Natural Sciences and Engineering Research Council of Canada (NSERC) under Strategic Projects. The first author gratefully acknowledges financial supports from the School of Graduate Studies, Concordia University.

The authors are with the Department of Electrical and Computer Engineering, Faculty of Engineering and Computer Science, Concordia University, 1455 de Maisonneuve Blvd. West, Montreal, Quebec, Canada H3G 1M8. The second author is also with the Canadian Space Agency (CSA), Saint-Hubert, QC, Canada. Emails: armehrabian@ieee.org, tafazoli@ieee.org, and kash@ece.concordia.ca. 
applications to spacecraft (SC) formation flying missions and tele-operations, among others. The area of cooperative control has recently attracted great interest and attention by researchers. A substantial body of work has already appeared in the literature. However, in many of these works the emphasis has been focused on merely a specific class of systems, such as single or double integrator systems [1], [2], linear systems [3], [4], UAVs [5], [6], [7], or robot manipulators [8], [9], [10], [11], [12], [13], among others.

The reported results in [8], [9] rely only on linear interconnections among the agents for synchronization and trajectory tracking objectives. Output and state synchronization of a network of passive systems is considered in [14]. However, for state synchronization of multiple robot networks, the control law proposed in [14] requires the knowledge of the inertia matrix and the Coriolis and centrifugal forces/torques. Furthermore, the bound on the control efforts in [8], [9], and [14] depends on the selection of the initial conditions (that is, the initial set-point tracking errors). In other words, the larger one selects the initial conditions, the larger the bound on the control effort will be. Furthermore, in [10] the authors have addressed consensus seeking problem (and not set-point tracking) among a class of robot manipulators.

In real life systems, actuators are physically constrained and are generally subject to faults and failures (permanent or intermittent). This requires that the selected and designed controllers should be fault-tolerant and reconfigurable. In other words, in presence of actuator faults, the controller should be able to maintain its minimum control objectives by reconfiguring its own structure (or by changing its gains) so that degradations in the overall system performance can be managed and handled efficiently and effectively. Reconfigurable control systems have been investigated extensively in the literature for the past three decades. A bibliographical review of this area can be found in [15].

The main objective of this paper is to present a switching-based control reconfiguration strategy that is utilized in case of an actuator fault or a saturation constraint to accomplish cooperative control of (electro-) mechanical EulerLagrange (EL) systems. Towards this end, we first introduce a class of distributed controllers (denoted as nominal) that can be used for accomplishing cooperative state synchronization and set-point tracking. This will be achieved under a fixed network topology consisting of potentially multiple leaders and followers and without requiring any knowledge of the inertia matrix and the Coriolis and centrifugal forces/torques of the agents. It is shown that by using a special class of our proposed distributed controllers, boundedness of the closed-loop networked EL system states and control efforts are guaranteed regardless of the initial conditions. We then introduce a class of distributed constrained controllers (denoted as reconfigured) that can be used to maintain the overall control objectives of the EL system in presence of actuator faults and constraints. Finally, we introduce a procedure that can be employed to switch between the two distributed controllers (the nominal and the reconfigured controllers). In presence of actuator faults and saturations, a switching mechanism is introduced to provide a reconfigurable controller for the networked EL system.

The outline of the remainder of the paper is as follows. In Section II, a brief overview of the necessary assumptions and preliminaries are presented. Section III, presents the proposed cooperative control of networked EL systems with and without actuator constraints. The proposed reconfigurable cooperative control of the networked EL system 
is presented in Section IV. Simulation results for the problem of cooperative control of a team of robot manipulators subject to an actuator fault and saturation constraint are presented in Section $\mathrm{V}$ to demonstrate and illustrate the advantages and benefits of the proposed reconfigurable control strategy. Finally, conclusions are stated in Section VI.

\section{Assumptions AND PRELIMINARies}

\section{A. Euler-Lagrange Systems}

Dynamics of the class of (electro-) mechanical systems that are considered in this work are described by the Euler-Lagrange (EL) equations of motion. Examples of mechanical EL systems are UGVs, UAVs, SC, among others. Examples of electro-mechanical EL systems include magnetic levitation systems, AC machines, among others [16]. An advantage of the EL formulation is that the dynamical equations are independent of the coordinate system that is used. It is assumed, in this paper, that the $j$-th EL system (agent) has the following dynamics, namely,

$$
D_{j}\left(q_{j}\right) \ddot{q}_{j}+C_{j}\left(q_{j}, \dot{q}_{j}\right) \dot{q}_{j}+g_{j}\left(q_{j}\right)+\frac{\partial \mathscr{F}_{j}\left(\dot{q}_{j}\right)}{\partial \dot{q}_{j}}=M_{j} u_{j}
$$

where $q_{j} \in \mathbb{R}^{k}$ denotes the generalized coordinates, $D_{j}\left(q_{j}\right) \in \mathbb{R}^{k \times k}$ denotes a symmetric positive definite matrix known as the general inertia matrix, $C_{j}\left(q_{j}, \dot{q}_{j}\right) \dot{q}_{j}$ denotes the Coriolis and centrifugal forces, and $g_{j}\left(q_{j}\right)$ denotes as the gravitational force vector (GFV). Moreover, $\mathscr{F}_{j}\left(\dot{q}_{j}\right)$ denotes the Rayleigh dissipation function, which by definition satisfies $\dot{q}_{j}^{T} \frac{\partial \mathscr{F}_{j}\left(\dot{q}_{j}\right)}{\partial \dot{q}_{j}} \geq 0$, and $\dot{q}_{j} \equiv 0$ implies $\frac{\partial \mathscr{F}_{j}\left(\dot{q}_{j}\right)}{\partial \dot{q}_{j}}=0$. It is further assumed that the control signal $u_{j} \in \mathbb{R}^{k}$ enters into the EL system dynamics linearly, and $M_{j} \in \mathbb{R}^{k \times k}$ is a nonsingular constant matrix.

The dynamical model (1) enjoys the following properties [16], namely, P1: The general inertia matrix is bounded, specifically, $\exists \underline{k}_{j}, \bar{k}_{j}$ such that: $\underline{k}_{j} \mathfrak{I}_{k}<D_{j}\left(q_{j}\right)<\bar{k}_{j} \mathfrak{I}_{k}, \forall q_{j}$, where $\mathfrak{I}_{k}$ is an $k \times k$ identity matrix, P2: GFV is assumed to be upper bounded, that is, $0 \leq \sup _{q_{j} \in \Re^{k}}\left\{\left|g_{i, j}\left(q_{j}\right)\right|\right\} \leq \bar{g}_{i, j}, \forall i \in\{1, \ldots, k\}$, where $g_{i, j}\left(q_{j}\right)$ denotes the elements of $g_{j}\left(q_{j}\right)$, and P3: $\dot{D}_{j}\left(q_{j}\right)-2 C_{j}\left(q_{j}, \dot{q}_{j}\right)$ is a skew-symmetric matrix.

We now introduce a notion of the saturation function that will be used subsequently.

Definition 1. A saturation function denoted by $S a t(x): \mathbb{R} \rightarrow \mathbb{R}$, is an odd function with the following properties $\forall x \in \mathbb{R}$, namely, (i) $\operatorname{Sat}(x)=0$, if and only if $x=0$; (ii) $|\operatorname{Sat}(x)| \leq 1$; (iii) $\operatorname{Sat}(-x)=-\operatorname{Sat}(x)$; (iv) $\frac{\partial \operatorname{Sat}(x)}{\partial x} \geq 0$ and $\frac{\partial \operatorname{Sat}(x)}{\partial x} \neq 0$, when $x=0$; and (v) there exists a constant $b>0$ such that $\forall x \in[-b, b]$, we have $\operatorname{Sat}(x)=\gamma x$, where $\gamma>0$

There are several functions that globally satisfy the above requirements. Examples include $\tanh \left(\kappa^{2} x\right)$, and the function $\frac{x}{\sqrt{\kappa^{2}+x^{2}}}, \forall x \in \mathbb{R}$ with a constant parameter $\kappa$, to name a few. It can be shown that the saturation function satisfying the conditions of Definition 1 enjoys the property $\int_{0}^{x_{1}} \operatorname{Sat}(x) d x \geq \frac{1}{2} \operatorname{Sat}\left(x_{1}\right) x_{1} \geq 0, \forall x, x_{1} \in \mathbb{R}[16]$.

\section{B. Graph Theory and the Communication Topology}

In this paper, it is assumed that information exchanges among the ' $m$ ' EL systems (agents) can be represented by a graph $\mathscr{G}$. Graph $\mathscr{G}$ consists of a node set $\mathscr{V}=\{1, \ldots, m\}$, an edge set $\mathscr{E} \subseteq \mathscr{V} \times \mathscr{V}$, and a weighted adjacency 
matrix $\Lambda=\left[\lambda_{j n}\right] \in \Re^{m \times m}$. The $m$ agents in the network are considered as nodes of the graph $\mathscr{G}$. The communication links among the agents are considered as the graph edge set.

The weighted adjacency matrix $\Lambda$ is defined such that $\lambda_{j n}=\lambda_{n j}$ is a positive weight if $(j, n) \in \mathscr{E}$, while $\lambda_{j n}=\lambda_{n j}=$ 0 , otherwise. Associated with $\Lambda$, we introduce a symmetric positive semi-definite matrix known as the Laplacian matrix $\mathscr{L}=\left[l_{j n}\right] \in \mathfrak{R}^{m \times m}$, such that $l_{j j}=\sum_{n=1, n \neq j}^{m} \lambda_{j n}$ and $l_{j n}=-\lambda_{j n}$, where $k \neq j$. Furthermore, if the graph is connected, $\mathscr{L}$ has a simple eigenvalue 0 with an associated eigenvector of $\mathbf{1}_{m}$, where $\mathbf{1}_{m}$ denotes an $m \times 1$ column vector of ones. All the other eigenvalues of $\mathscr{L}$ are positive if and only if the graph $\mathscr{G}$ is connected.

The edge count of the graph $\mathscr{G}$, denoted by $|\mathscr{E}(\mathscr{G})|$, is the number of edges in the graph $\mathscr{G}$. For a given node $j$ in the communication graph, the set of agents from which it can receive information is called a neighboring set $\mathscr{N}_{j}$, that is $\forall j=1, \ldots, n: \mathscr{N}_{j}=\{n=1, \ldots, m \mid(j, n) \in \mathscr{E}\}$. In addition, the number of the $j$-th agent neighbors is denoted by $\left|\mathscr{N}_{j}\right|$ (known as the cardinality of the set $\mathscr{N}_{j}$ ). We are now in a position to state our first Assumption.

Assumption 1. Throughout the paper it is assumed that the communication graph is strongly connected (bidirectional).

Some examples of the communication graphs that can be considered in this paper corresponding to for example, 3, 4, 6 and 8 agents in the network as per Assumption 1 are shown in Fig. 1. The communication graphs shown in this figure are bi-directionally connected and with minimum number of edges.

\section{Cooperative Control of Networked Lagrangian Systems With and Without Actuator}

\section{CONSTRAINTS}

We start this section by introducing the notions of state synchronization and set-pint tracking errors. The problem definition and requirements are then presented. Next, we develop and present distributed cooperative controllers that are with and without actuator constraints. This will be followed by some preliminary results. Finally, the main result of this section is presented in a theorem.

\section{A. State Synchronization and Set-Point Tracking Errors}

In this section, a strategy for coordinated control of a network of multi-agent EL systems is developed. Let us denote the desired coordinates for the $j$-th EL system (agent) by $q_{j}^{\star}$. The set-point tracking error between the coordinates of the $j$-th agent and its desired coordinates is defined according to

$$
\delta q_{j}=q_{j}-q_{j}^{\star}, \quad j \in \mathscr{V}
$$

It is assumed that the desired coordinates $q_{j}^{\star}$ are set to constant values. Therefore, we have $\delta \dot{q}_{j}=\dot{q}_{j}, \quad j \in \mathscr{V}$. Moreover, the synchronization error between the coordinates of the $j$-th and the $n$-th EL systems and its time derivative are defined according to

$$
q_{j n}=\delta q_{j}-\delta q_{n}, \quad \dot{q}_{j n}=\dot{q}_{j}-\dot{q}_{n}
$$

Note that as long as $q_{j}^{\star}-q_{n}^{\star} \neq 0$, the agents are assumed to not collide with one another at the steady-state. 


\section{B. Problem Definition and Requirements}

The objective of this section is to introduce a distributed cooperative control law for the $j$-th (electro-) mechanical EL system. This distributed control law has to guarantee the following requirements, namely: (1) the closed-loop networked system's states and control signals remain globally stable, (2) the synchronization errors asymptotically converge to origin, i.e., $q_{j n} \rightarrow 0$ and $\dot{q}_{j n} \rightarrow 0$ as $t \rightarrow \infty$ (this is designated as the station-keeping behavior), and (3) the set-point tracking errors asymptotically converge to origin, i.e., $\delta q_{j} \rightarrow 0, \dot{q}_{j} \rightarrow 0$ as $t \rightarrow \infty$ (this is designated as the formation-keeping behavior). We also consider actuator saturation constraints in the design of the distributed cooperative control law for the $j$-th EL system, which will be presented formally subsequently.

\section{The Distributed Cooperative Control Design}

The distributed cooperative control law considered in this paper has two main parts: (i) The station-keeping control, and (ii) the formation-keeping control. The station-keeping control is introduced to satisfy the first and the second objectives of the coordinated control of the EL system. The formation-keeping control is introduced to satisfy the first and the third objectives of the coordinated control of the EL system.

As opposed to the centralized multi-input multi-output (MIMO) cooperative control laws (cf. [17]), our proposed control laws do not require knowledge of all the agents states in the network. In other words, our proposed distributed control laws only require information from their own agent states and their neighboring agents states in the network. Due to the dependency of the control command of the $j$-th agent on the states of its neighboring agents $\mathscr{N}_{j}$, the proposed control law cannot be considered "strictly" as a decentralized controller, where the control command only depends on the agent's own states (cf. [18]).

We therefore introduce the following distributed nominal nonlinear cooperative control for the $j$-th EL system to satisfy the three objectives introduced in the previous subsection simultaneously, namely,

$$
u_{j}=M_{j}^{-1}(\underbrace{-\Lambda_{j}^{p} \chi\left(\delta q_{j}\right)-\Lambda_{j}^{d} \chi\left(\dot{q}_{j}\right)+g_{j}\left(q_{j}\right)}_{\text {station-keeping control }}-\underbrace{\sum_{n \in \mathscr{N}_{j}} \Lambda_{j n}^{p} \chi\left(q_{j n}\right)-\sum_{n \in \mathscr{N}_{j}} \Lambda_{j n}^{d} \chi\left(\dot{q}_{j n}\right)}_{\text {formation-keeping control }})
$$

where $\chi(\mathbf{x})=\operatorname{col}\left[\chi\left(x_{1}\right), \ldots, \chi\left(x_{n}\right)\right]$, where $\chi(x)$ is a monotonically increasing odd function. In addition, the control gains $\Lambda_{j}^{p} \succeq 0, \Lambda_{j n}^{p}=\Lambda_{n j}^{p} \succ 0, \Lambda_{j}^{d} \succeq 0$, and $\Lambda_{j n}^{d}=\Lambda_{n j}^{d} \succ 0$ are diagonal matrices of (positive) proportional and derivative gains (superscript ' $p$ ' denotes proportional and superscript ' $d$ ' denotes derivative). The first three terms of the above control law are also called the station-keeping control. The last two terms of the above control law are called the formation-keeping control.

In practice, it is not always possible to provide the desired coordinates to all the EL system agents in the network. The agents that receive the desired coordinates can be considered as leaders. Therefore, for the team leaders we have $\Lambda_{j}^{p} \succ 0$ and $\Lambda_{j}^{d} \succ 0$. The agents that do not receive the desired coordinates can be considered as followers. Consequently, for the team followers we have $\Lambda_{j}^{p}=0$ and $\Lambda_{j}^{d}=0$. To guarantee strong connectivity of the communication graph for the followers there should exist an $n \in\{1, \ldots, m\}, n \neq j$, such that $\Lambda_{j n}^{p}, \Lambda_{j n}^{d} \neq 0$. In 
other words, every EL system agent in the communication graph only needs to be connected (bidirectional) to a leader. We now formally state our next assumption below.

Assumption 2. The following conditions are assumed to hold for the considered EL systems:

a. The EL systems are fully actuated at all times. This implies that the dimension of the input vector is equal to the dimension of the generalized coordinates vector at all times,

b. The maximum control effort available to each actuator is constrained at all times due to the inherent physical saturation constraints. Specifically, the maximum control effort for the $i$-th actuator of the $j$-th EL system, in absence of faults, is constrained by $\left.\bar{u}_{i}^{\max }\right|_{j}$,

c. In presence of actuator faults, the maximum control effort for the $i$-th actuator of the $j$-th EL system may fall below $\left.\bar{u}_{i}^{\max }\right|_{j}$ and this maximum bound could be time-varying, in general, and is denoted by $\left.u(t)_{i}^{\max }\right|_{j}$,

d. The maximum control effort available in presence of the worst-case actuator faults, $\left.u(t)_{i}^{\max }\right|_{j}$, is bounded from below. Specifically, the least upper bound of the available control effort for the $i$-th actuator of the $j$-th EL system under all possible faults and constraints is known a priori and is denoted by $\left.\underline{u}_{i}^{\max }\right|_{j}$,

e. The above control bounds satisfy, $0 \leq\left\|M_{j}^{-1} g_{j}\left(q_{j}\right)\right\|<\left\|\underline{u}_{j}^{\max }\right\| \leq\left\|u(t)_{j}^{\max }\right\|<\left\|\bar{u}_{j}^{\max }\right\|$. This implies that the actuators should maintain the $j$-th system at rest corresponding to all desired positions.

We first present the following lemma, which is used subsequently in the paper.

Lemma 1. Consider the following algebraic equations that correspond to a strongly connected network of ' $m$ ' agents

$$
\Lambda_{j}^{p} \chi\left(\delta q_{j}\right)+\sum_{n \in \mathscr{N}_{j}} \Lambda_{j n}^{p} \chi\left(q_{j n}\right)=0, \quad j, n \in\{1, \ldots, m\}, j \neq n
$$

where $\delta q_{j} \in \mathbb{R}^{k}, \chi(x)$ is a monotonically increasing odd function, and $\Lambda_{j n}^{p}=\Lambda_{n j}^{p}$ are positive definite matrices. Furthermore, assume that $\Lambda_{j}^{p}$ is a positive definite diagonal matrix for only $0<l \leq m$ number of equations (corresponding to ' $l$ ' leaders) and is zero, otherwise. If we have $\sum_{j=1}^{l} \Lambda_{j}^{p} \chi\left(\delta q_{j}\right)=0$, then the only solution to (5) is $\delta q_{j}=0, \forall j \in\{1, \ldots, m\}$.

Proof: We prove this lemma by contradiction. First note that (5) implies that if for the $j$-th algebraic equation we have $\Lambda_{j}^{p}=0$ (corresponding to $m-l \geq 0$ followers), then $\sum_{n \in \mathscr{N}_{j}} \Lambda_{j n}^{p} \chi\left(q_{j n}\right)=0$. Therefore, (5) essentially reduces to $\Lambda_{j}^{p} \chi\left(\delta q_{j}\right)+\sum_{n \in \mathscr{N}_{j}} \Lambda_{j n}^{p} \chi\left(q_{j n}\right)=0, j, n \in\{1, \ldots, l\}, j \neq n$. Now let us assume that the claim does not hold, i.e. $\delta q_{j} \neq 0, \forall j \in\{1, \ldots, l\}$. This in view of $\sum_{j=1}^{l} \Lambda_{j}^{p} \chi\left(\delta q_{j}\right)=0$, implies that there exists at least one system (let's say the $l$-th system, without loss of any generality) for which we have: $\sum_{j=1}^{l-1} \Lambda_{j}^{p} \chi\left(\delta q_{j}\right)=-\Lambda_{l}^{p} \chi\left(\delta q_{l}\right) \equiv \Lambda_{l}^{p} \chi\left(-\delta q_{l}\right)$, which implies that the sign of the $l$-th system error is opposite to that of the others in the network. Without loss of generality, let us assume $\delta q_{l}=-\varepsilon_{j} \delta q_{j}, j=1, \ldots, l-1$, where $\varepsilon_{j}>0$, and that $\delta q_{j}>0, j=1, \ldots, l-1$. Thus, from (5) we have: $\Lambda_{l}^{p} \chi\left(\delta q_{l}\right)+\Lambda_{l, 1}^{p} \chi\left(\delta q_{l}-\delta q_{1}\right)+\Lambda_{l, 2}^{p} \chi\left(\delta q_{l}-\delta q_{2}\right)+\cdots+\Lambda_{l, l-1}^{p} \chi\left(\delta q_{l}-\delta q_{l-1}\right)=0$, which can be re-written as: $-\Lambda_{l}^{p} \chi\left(\varepsilon_{1} \delta q_{1}\right)-\Lambda_{l, 1}^{p} \chi\left[\left(\varepsilon_{1}+1\right) \delta q_{1}\right]-\cdots-\Lambda_{l, l-1}^{p} \chi\left[\left(\varepsilon_{l-1}+1\right) \delta q_{l-1}\right]=0$. The statement above does not hold when $\delta q_{j} \neq 0, \forall j \in\{1, \ldots, l\}$, which is a contradiction. Therefore, the only solution to the problem is to 
have $\delta q_{j}=0, j=1, \ldots, l$. Consequently, from (5) we have $\sum_{n \in \mathscr{N}_{j}} \Lambda_{j n}^{p} \chi\left(q_{j n}\right)=0, \forall j, n \in\{1, \ldots, m\}, j \neq n$, which by the strong connectivity of the communication graph, and the fact that $\chi(x)$ is a monotonically increasing odd function implies $q_{j n}=0, \forall j, n \in\{1, \ldots, m\}, j \neq n$. Consequently, in view of (3), one obtains $\delta q_{j}=0, j=l+1, \ldots, m$. Therefore, we have $\delta q_{j}=0, \forall j \in\{1, \ldots, m\}$. This completes the proof of the lemma.

We are now in a position to present the main result of this section.

\section{Main Result}

Our main result in this subsection is provided in the following theorem.

Theorem 1. Consider a network of ' $m$ ' multiple heterogeneous (electro-) mechanical EL systems where the $j$ th agent's dynamics is governed by (1). Without loss of generality, let us assume that agent 1 to agent ' $l$ ' are the network leaders and agent ' $l+l$ ' to agent ' $m$ ' are the followers. Furthermore, consider that the distributed "nominal" cooperative control law for the $j$-th agent is selected according to equation (4). Then under Assumption 1 and Assumption 2 (parts (a) and (e)) it is guaranteed that: (i) all the signals of the closed-loop EL system will remain bounded, (ii) the synchronization errors asymptotically converge to origin, i.e., $q_{j n} \rightarrow 0$ and $\dot{q}_{j n} \rightarrow 0$ as $t \rightarrow \infty$, for all $j \in \mathscr{V}, n \in \mathscr{N}_{j}$, and, (iii) the set-point tracking errors converge asymptotically to origin, i.e., $\delta q_{j} \rightarrow 0$, $\dot{q}_{j} \rightarrow 0$ as $t \rightarrow \infty$ for all $j \in \mathscr{V}$. Furthermore, suppose that one sets $\chi(x) \triangleq \operatorname{Sat}(x)$ in the control law (4) and let the gains of the $j$-th EL system satisfy the following inequalities,

$$
\left.\bar{m}_{i}\right|_{j}\left(\left.\lambda_{i}^{p}\right|_{j}+\left.\lambda_{i}^{d}\right|_{j}+\left.\sum_{n \in \mathscr{N}_{j}} \lambda_{i}^{p}\right|_{j n}+\left.\sum_{n \in \mathscr{N}_{j}} \lambda_{i}^{d}\right|_{j n}+\left.g_{i}\right|_{j}\right) \leq\left.\bar{u}_{i}^{\max }\right|_{j}, i \in\{1, \ldots, k\}, j \in\{1, \ldots, m\}
$$

where $\bar{m}_{i}$ denotes the $i$-th element of the $M_{j}^{-1}$ matrix, $\left.\lambda_{i}^{\sigma}\right|_{j}, \sigma=p, d$ denotes the $i$-th element of the $\Lambda_{j}^{\sigma}$ matrix and $\left.\lambda_{i}^{\sigma}\right|_{j n}, \sigma=p, d$ denotes the $i$-th element of the $\Lambda_{j n}^{\sigma}$ matrix. Then, under Assumption 1 and Assumption 2 (parts $(a),(b)$, and (e)) in addition to guaranteeing conditions (i)-(iii) above, one can also ensure that, (iv) the actuator constraints are satisfied, that is $\left|u(t)_{i}\right|_{j} \mid \leq \bar{u}_{i}^{\max }{ }_{j}, \forall j \in\{1, \ldots, m\}, \forall t \geq 0$ and for all initial conditions.

Proof: In order to prove the theorem, let us introduce the following positive definite radially unbounded Lyapunov function candidate for the network of EL systems

$$
\mathscr{W}\left(\delta q_{j}, \dot{q}_{j}, q_{j n}\right)=\sum_{j=1}^{m}\left(\frac{1}{2} \dot{q}_{j}^{T} D_{j}\left(q_{j}\right) \dot{q}_{j}+\left.\sum_{i=1}^{k} \lambda_{i}^{p}\right|_{j} \int_{0}^{\delta q_{i, j}} \chi(x) d x+\left.\frac{1}{2} \sum_{n \in \mathscr{N}_{j}} \sum_{i=1}^{k} \lambda_{i}^{p}\right|_{j n} \int_{0}^{q_{i, j n}} \chi(x) d x\right)
$$

where $\left.\lambda_{i}^{p}\right|_{j}$ denotes the $i$-th diagonal element of $\Lambda_{j}^{p}$ and $\left.\lambda_{i}^{p}\right|_{j n}$ denotes the $i$-th diagonal element of $\Lambda_{j n}^{p}$. The time derivative of the above Lyapunov function candidate, by taking into account the expressions (2) and (3), is given by

$$
\dot{\mathscr{W}}=\sum_{j=1}^{m}\left(\dot{q}_{j}^{T} D_{j}\left(q_{j}\right) \ddot{q}_{j}+\frac{1}{2} \dot{q}_{j}^{T} \dot{D}_{j}\left(q_{j}\right) \dot{q}_{j}+\dot{q}_{j}^{T} \Lambda_{j}^{p} \chi\left(\delta q_{j}\right)+\frac{1}{2} \sum_{n \in \mathscr{N}_{j}} \dot{q}_{j n}^{T} \Lambda_{j n}^{p} \chi\left(q_{j n}\right)\right)
$$


Note that the communication links are bidirectional (as per Assumption 1), therefore one can show that

$$
\begin{aligned}
\frac{1}{2} \sum_{j=1}^{m} \sum_{n \in \mathscr{N}_{j}} \dot{q}_{j n}^{T} \Lambda_{j n}^{p} \chi\left(q_{j n}\right) & =\sum_{j=1}^{m} \sum_{n \in \mathscr{N}_{j}} \dot{q}_{j}^{T} \Lambda_{j n}^{p} \chi\left(q_{j n}\right) \\
\sum_{j=1}^{m} \dot{q}_{j}^{T} \sum_{n \in \mathscr{N}_{j}} \Lambda_{j n}^{d} \chi\left(\dot{q}_{j n}\right) & =\sum_{j=1}^{m} \sum_{n \in \mathscr{N}_{j}} \dot{q}_{j n}^{T} \Lambda_{j n}^{d} \chi\left(\dot{q}_{j n}\right)
\end{aligned}
$$

By noting the skew-symmetric property of the matrix $\dot{D}_{j}\left(q_{j}\right)-2 C_{j}\left(q_{j}, \dot{q}_{j}\right)$ (cf. property P3), and the fact that for the followers we have $\Lambda_{j}^{p}=\Lambda_{j}^{d}=0$, and by using the governing dynamics (1) and (4) one can then yield

$$
\begin{aligned}
\dot{\mathscr{W}}= & \sum_{j=1}^{m}\left[\dot{q}_{j}^{T}\left(-C_{j}\left(q_{j}, \dot{q}_{j}\right) \dot{q}_{j}-g_{j}\left(q_{j}\right)-\frac{\partial \mathscr{F}_{j}\left(\dot{q}_{j}\right)}{\partial \dot{q}_{j}}+M_{j} u_{j}\right)\right. \\
& \left.+\frac{1}{2} \dot{q}_{j}^{T} \dot{D}_{j}\left(q_{j}\right) \dot{q}_{j}+\dot{q}_{j}^{T} \Lambda_{j}^{p} \chi\left(\delta q_{j}\right)+\frac{1}{2} \sum_{n \in \mathscr{N}_{j}} \dot{q}_{j n}^{T} \Lambda_{j n}^{p} \chi\left(q_{j n}\right)\right] \\
= & -\sum_{j=1}^{m}\left[\dot{q}_{j}^{T} \frac{\partial \mathscr{F}_{j}\left(\dot{q}_{j}\right)}{\partial \dot{q}_{j}}+\dot{q}_{j}^{T} \Lambda_{j}^{p} \chi\left(\delta q_{j}\right)+\frac{1}{2} \sum_{n \in \mathscr{N}_{j}} \dot{q}_{j n}^{T} \Lambda_{j n}^{p} \chi\left(q_{j n}\right)\right] \\
& +\sum_{j=1}^{m} \dot{q}_{j}^{T}\left(-\Lambda_{j}^{p} \chi\left(\delta q_{j}\right)-\Lambda_{j}^{d} \chi\left(\dot{q}_{j}\right)-\sum_{n \in \mathscr{N}_{j}} \Lambda_{j n}^{p} \chi\left(q_{j n}\right)-\sum_{n \in \mathscr{N}_{j}} \Lambda_{j n}^{d} \chi\left(\dot{q}_{j n}\right)\right) \\
= & -\sum_{j=1}^{l} \dot{q}_{j}^{T} \Lambda_{j}^{d} \chi\left(\dot{q}_{j}\right)-\sum_{j=1}^{m} \dot{q}_{j}^{T} \frac{\partial \mathscr{F}_{j}\left(\dot{q}_{j}\right)}{\partial \dot{q}_{j}}-\sum_{j=1}^{m} \sum_{n \in \mathscr{N}_{j}} \dot{q}_{j n}^{T} \Lambda_{j n}^{d} \chi\left(\dot{q}_{j n}\right) \leq 0
\end{aligned}
$$

which is a negative semi-definite decrescent function. First note that since the Lyapunov function is radially

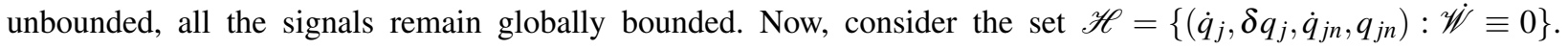
When $\dot{\mathscr{W}} \equiv 0$ ( $\dot{\mathscr{W}}$ is identically equal to zero), we have $\ddot{q}_{j}=\dot{q}_{j}=\dot{q}_{j n}=0$, therefore, the closed-loop dynamics of the $j$-th EL system can be written as $D_{j}\left(q_{j}\right) \ddot{q}_{j}=-\Lambda_{j}^{p} \chi\left(\delta q_{j}\right)-\sum_{n \in \mathscr{N}_{j}} \Lambda_{j n}^{p} \chi\left(q_{j n}\right)=0$. By noting the fact that $\Lambda_{j n}^{p}=\Lambda_{n j}^{p}$ and $\chi\left(q_{j n}\right)=-\chi\left(q_{n j}\right)$, it is straightforward to show that $\sum_{j=1}^{m} \sum_{n \in \mathscr{N}_{j}} \Lambda_{j n}^{p} \chi\left(q_{j n}\right)=0$, which implies $\sum_{j=1}^{l} \Lambda_{j}^{p} \chi\left(\delta q_{j}\right)=0$. Therefore, the requirements of Lemma 1 are satisfied, and one can conclude that $\delta q_{j}=q_{j n}=0, \forall j, n \in\{1, \ldots, m\}, j \neq n$. Therefore, the largest invariant set in $\mathscr{H}$ is the origin. Thus, by invoking the LaSalle's invariance theorem [19], and due to the fact that $\mathscr{W}$ is a positive definite radially unbounded function, it follows under Assumption 2 (parts (a) and (e)) that: (i) the states and control signals of the closed-loop networked EL system are globally stable, (ii) the synchronization errors asymptotically converge to origin, i.e., $q_{j n} \rightarrow 0$ and $\dot{q}_{j n} \rightarrow 0$ as $t \rightarrow \infty$, and, (iii) the set-point tracking errors asymptotically converge to origin, i.e., $\delta q_{j} \rightarrow 0, \dot{q}_{j} \rightarrow 0$ as $t \rightarrow \infty$.

By noting the fact that $|\operatorname{Sat}(x)| \leq 1, \forall x \in \mathbb{R}$ (cf. Definition 1), it is straightforward to show that by setting $\chi(x) \triangleq S a t(x)$ in the control law (4), and provided that the conditions (6) are satisfied, one has $\left|u(t)_{i}\right|_{j}\left|\leq \bar{u}_{i}^{\max }\right|_{j}, \forall j \in$ $\{1, \ldots, m\}, \forall t \geq 0$ and for all initial conditions. Therefore, under Assumption 2 (parts (a), (b) and (e)), it now follows that the properties (i)-(iii) above hold, and moreover the property (iv), that is the satisfaction of the actuator constraints also hold, namely, $\left|u(t)_{i}\right|_{j}\left|\leq \bar{u}_{i}^{\max }\right|_{j}, \forall j \in\{1, \ldots, m\}, \forall t \geq 0$ and for all initial conditions. This completes the proof of the theorem.

It is important to note that our proposed nominal distributed controller (4) is model-independent, in the sense 
that it does not require any information on the system's inertia matrix $D_{j}\left(q_{j}\right)$ as well as $C_{j}\left(q_{j}, \dot{q}_{j}\right)$. The controller requires only measurements from the coordinates of the system and information on the GFV.

\section{Reconfigurable Cooperative Control of Networked Lagrangian Systems}

In the previous section, we introduced a distributed approach for cooperative control of networked EL systems. It was shown that by using a special class of our proposed nominal distributed control approach (that is, by setting $\chi(x)$ to a $S a t(x)$ function), the upper bound on the control effort does not depend on the initial conditions. We are now in a position to present the following definitions.

Definition 2. A distributed controller is called nominal if the control law is given by

$$
u_{j}^{n o m}=M_{j}^{-1}\left(-\bar{\Lambda}_{j}^{p} \chi\left(\delta q_{j}\right)-\bar{\Lambda}_{j}^{d} \chi\left(\dot{q}_{j}\right)+g_{j}\left(q_{j}\right)-\sum_{n \in \mathscr{N}_{j}} \bar{\Lambda}_{j n}^{p} \chi\left(q_{j n}\right)-\sum_{n \in \mathscr{N}_{j}} \bar{\Lambda}_{j n}^{d} \chi\left(\dot{q}_{j n}\right)\right)
$$

where 'nom' stands for nominal, $\chi(x) \triangleq \operatorname{Sat}_{1}(x)\left(\operatorname{Sat}_{1}(x)\right.$ is a saturation function defined according to Definition 1), and the controller gains are $\bar{\Lambda}_{j}^{\sigma}=\operatorname{diag}\left(\left.\bar{\lambda}_{1}^{\sigma}\right|_{j}, \ldots,\left.\bar{\lambda}_{k}^{\sigma}\right|_{j}\right)$ and $\bar{\Lambda}_{j n}^{\sigma}=\operatorname{diag}\left(\left.\bar{\lambda}_{1}^{\sigma}\right|_{j n}, \ldots,\left.\bar{\lambda}_{k}^{\sigma}\right|_{j n}\right)$, where $\sigma=p, d$, such that the following constrained conditions are satisfied

$$
\left.\bar{m}_{i}\right|_{j}\left(\left.\bar{\lambda}_{i}^{p}\right|_{j}+\left.\bar{\lambda}_{i}^{d}\right|_{j}+\left.\sum_{n \in \mathscr{N}_{j}} \bar{\lambda}_{i}^{p}\right|_{j n}+\left.\sum_{n \in \mathscr{N}_{j}} \bar{\lambda}_{i}^{d}\right|_{j n}+\left.g_{i}\right|_{j}\right) \leq\left.\underline{u}_{i}^{\max }\right|_{j}, i \in\{1, \ldots, k\}, j \in\{1, \ldots, m\}
$$

under the nominal EL system operations.

Definition 3. A distributed controller is called reconfigured if the control law is given by

$$
u_{j}^{r f g}=M_{j}^{-1}\left(-\underline{\Lambda}_{j}^{p} \chi\left(\delta q_{j}\right)-\underline{\Lambda}_{j}^{d} \chi\left(\dot{q}_{j}\right)+g_{j}\left(q_{j}\right)-\sum_{n \in \mathscr{N}_{j}} \underline{\Lambda}_{j n}^{p} \chi\left(q_{j n}\right)-\sum_{n \in \mathscr{N}_{j}} \underline{\Lambda}_{j n}^{d} \chi\left(\dot{q}_{j n}\right)\right)
$$

where 'rfg' stands for reconfigured, $\chi(x) \triangleq \operatorname{Sat}_{2}(x)\left(\operatorname{Sat}_{2}(x)\right.$ is a saturation function defined according to Definition 1) and the controller gains are $\underline{\Lambda}_{j}^{\sigma}=\operatorname{diag}\left(\left.\underline{\lambda}_{1}^{\sigma}\right|_{j}, \ldots,\left.\underline{\lambda}_{k}^{\sigma}\right|_{j}\right)$ and $\underline{\Lambda}_{j n}^{\sigma}=\operatorname{diag}\left(\left.\underline{\lambda}_{1}^{\sigma}\right|_{j n}, \ldots,\left.\underline{\lambda}_{k}^{\sigma}\right|_{j n}\right)$, where $\sigma=p, d$, such that the following constrained conditions are satisfied

$$
\left.\bar{m}_{i}\right|_{j}\left(\left.\underline{\lambda}_{i}^{p}\right|_{j}+\left.\underline{\lambda}_{i}^{d}\right|_{j}+\left.\sum_{n \in \mathscr{N}_{j}} \underline{\lambda}_{i}^{p}\right|_{j n}+\left.\sum_{n \in \mathscr{N}_{j}} \underline{\lambda}_{i}^{d}\right|_{j n}+\left.g_{i}\right|_{j}\right) \leq\left.\underline{u}_{i}^{\max }\right|_{j}, i \in\{1, \ldots, k\}, j \in\{1, \ldots, m\}
$$

under the faulty EL system operations.

Note that the above definitions allow the functions $\operatorname{Sat}_{1}(x)$ and $\operatorname{Sat}_{2}(x)$ to be different. In other words, the functions $\operatorname{Sat}_{1}(x)$ and $\operatorname{Sat}_{2}(x)$ given in Definitions 2 and 3 are saturation functions with possibly different structures.

In presence of actuator faults, the maximum control effort available to each actuator may change (cf. Assumption 2 (d)). In this case, the nominal controller must be reconfigured in order to satisfy the constraints on the control effort due to the actuator faults. Note that these two controllers do not have the same structure and do not employ the same gains. The control reconfiguration is to be accomplished and achieved by switchings between the nominal and the reconfigured controllers.

Our goal in this section is to show that by any number of switchings between these two controllers it follows that: (a) a globally stable closed-loop EL system is obtained, (b) the synchronization errors asymptotically converge to 
zero, and (c) the tracking errors asymptotically converge to zero, provided that certain conditions are satisfied. These are very useful properties as they show that in case of a fault and presence of a subsequent actuator saturation constraint one can switch from the nominal controller to the reconfigured controller while still ensuring desirable behavior of the overall networked EL system. Furthermore, when the injected actuator fault is removed (corresponding to an intermittent fault), one can switch back from the Rreconfigured controller to the nominal controller. One of the advantages of this switching strategy is that the only information that is required for controller reconfiguration is the knowledge of the fault occurrence, which can be determined through a large body of fault detection algorithms that are available in the literature (refer to our recent works in [20], [21], [22] and references therein). This approach does not require exact knowledge of the severity of the fault (fault identification) as long as Assumption 2 (c)-(f) is satisfied. Furthermore, the requirements for the switching operation is rather straightforward to satisfy and implement in practice. We now state the following definition and assumption before presenting our main result of this section.

Definition 4. Let $\mathscr{W}_{1}(x)$ represent $\mathscr{W}(x)$ given by equation (8) with $\chi(x) \triangleq \operatorname{Sat}_{1}(x), \Lambda_{j}^{\sigma} \triangleq \bar{\Lambda}_{j}^{\sigma}$, and $\Lambda_{j n}^{\sigma} \triangleq \bar{\Lambda}_{j n}^{\sigma}, \sigma=$ $p, d$. Furthermore, let $\mathscr{W}_{2}(x)$ represent $\mathscr{W}(x)$ given by equation (8) with $\chi(x) \triangleq \operatorname{Sat}_{2}(x), \Lambda_{j}^{\sigma} \triangleq \underline{\Lambda}_{j}^{\sigma}$, and $\Lambda_{j n}^{\sigma} \triangleq$ $\underline{\Lambda}_{j n}^{\sigma}, \sigma=p, d$

Assumption 3. The following conditions are assumed to hold:

a. The controller gains and the saturation functions are selected such that $\bar{\Lambda}_{j}^{p} \operatorname{Sat}_{1}(x) \geq \underline{\Lambda}_{j}^{p} \operatorname{Sat}_{2}(x), \forall x$ and $\bar{\Lambda}_{j n}^{p} \operatorname{Sat}_{1}(x) \geq \underline{\Lambda}_{j n}^{p} \operatorname{Sat}_{2}(x), \forall x$. This from Definition 4 implies that $\mathscr{W}_{1}(x) \geq \mathscr{W}_{2}(x), \forall x$, where $x=\left[\dot{q}_{j}^{T}, \delta q_{j}^{T}, q_{j n}{ }^{T}\right]^{T}$. We denote $[-\bar{b}, \bar{b}]$ as the region where $\bar{\Lambda}_{j}^{p} \operatorname{Sat}_{1}(x)=\underline{\Lambda}_{j}^{p} \operatorname{Sat}_{2}(x)$ and $\bar{\Lambda}_{j n}^{p} \operatorname{Sat}_{1}(x)=\underline{\Lambda}_{j n}^{p} \operatorname{Sat}_{2}(x)$.

b. At every switching instant from the reconfigured controller to the nominal controller (this instant is at the designer's disposal since the fault is no longer present) one needs to ensure that $\delta q_{i, j}, q_{i, j n} \in[-\bar{b}, \bar{b}], i=$ $1, \ldots, k, j, n \in\{1, \ldots, m\}, j \neq n$ (existence of such a region is guaranteed in part (a)), whereas at the switching instant from the nominal controller to the reconfigured controller (this instant is not at the designer's disposal since the fault detection time is unknown) one cannot guarantee the size of $\delta q_{i, j}$ and $q_{i, j n}$.

c. The time between any two sequential switches can be arbitrarily small, however it should always be greater than a constant value $\bar{\tau}>0$. This can be interpreted as switchings with non-vanishing dwell-times [23].

We are now in a position to state the main result of this section.

Theorem 2. Consider a network of ' $m$ ' (electro-) mechanical EL systems where the $j$-th agent dynamics is governed by equation (1) and which is subject to the nominal distributed cooperative control law that is given by Definition 2. Also consider the same network and communication topology which is subject to the reconfigured distributed cooperative control law that is given by Definition 3. Any switchings between the nominal and the reconfigured closed-loop systems will yield a globally stable EL system and moreover, the state synchronization and the set-point tracking errors globally asymptotically converge to origin provided that the conditions of Assumptions 2 and 3 hold. 
Proof: Global asymptotic convergence of the state synchronization and the set-pint tracking errors, as well as global boundedness of all of the states and control signals of the closed-loop switched EL system under a finite number of switchings follow from Theorem 1. We consider an infinite number of switchings between the nominal system and the reconfigured system. We can pick an infinite subsequence of switching times from the reconfigured system to the nominal system, $t_{h_{1}}, t_{h_{2}}, \ldots$ and an infinite corresponding subsequence of switching times from the nominal system to the reconfigured system, $t_{h_{1}+1}, t_{h_{2}+1}, \ldots$ In view of condition (c) of Assumption 3 , the time between any two consecutive intervals, namely, $\left[t_{h_{e}}, t_{h_{e}+1}\right), e=1,2, \ldots$ is not less than $\bar{\tau}$ for the nominal system. We denote the union of these intervals by $\overline{\mathscr{E}}$. From the condition (v) of Definition 1, and by using the conditions (a) and (b) of Assumption 3, one can show that at each switching instant from the reconfigured controller to the nominal controller, we have $\left.\bar{\lambda}_{i}^{p}\right|_{j} \int_{0}^{\delta q_{i, j}} \operatorname{Sat}_{1}(\xi) d \xi=\left.\underline{\lambda}_{i}^{p}\right|_{j} \int_{0}^{\delta q_{i, j}} \operatorname{Sat}_{2}(\xi) d \xi$ and $\left.\bar{\lambda}_{i}^{p}\right|_{j n} \int_{0}^{q_{i, j n}} \operatorname{Sat} t_{1}(\xi) d \xi=$ $\left.\underline{\lambda}_{i}^{p}\right|_{j n} \int_{0}^{q_{i, j n}} \operatorname{Sat}_{2}(\xi) d \xi$. These properties along with the Definition 4 imply that at each switching instant from the reconfigured controller to the nominal controller we have $\mathscr{W}_{2}(\bar{y})=\mathscr{W}_{1}(\bar{y})$, where $\bar{y}=\left[\dot{q}_{j}^{T}, \delta q_{j}{ }^{T}, q_{j n}{ }^{T}\right]^{T}$.

Furthermore, from the condition (v) of Definition 1, and by using the conditions (a) and (b) of Assumption 3, one can show that at each switching instance from the nominal controller to the reconfigured controller, we have $\left.\bar{\lambda}_{i}^{p}\right|_{j} \int_{0}^{\delta q_{i, j}} \operatorname{Sat}_{1}(\xi) d \xi \geq\left.\underline{\lambda}_{i}^{p}\right|_{j} \int_{0}^{\delta q_{i, j}} \operatorname{Sat}_{2}(\xi) d \xi$ and $\left.\bar{\lambda}_{i}^{p}\right|_{j n} \int_{0}^{q_{i, j n}} \operatorname{Sat}_{1}(\xi) d \xi \geq\left.\underline{\lambda}_{i}^{p}\right|_{j n} \int_{0}^{q_{i, j n}} \operatorname{Sat}_{2}(\xi) d \xi$. These properties along with the Definition 4 imply that at each switching instant from the nominal controller to the reconfigured controller, we have $\mathscr{W}_{1}(\bar{y}) \geq \mathscr{W}_{2}(\bar{y})$. From equation (8), we have $\operatorname{Sgn}\left(\dot{\mathscr{W}}_{1}(y)\right)=\operatorname{Sgn}\left(\dot{\mathscr{W}}_{2}(y)\right)$, where $y=\left[\dot{q}_{j}^{T}, \dot{q}_{j n}^{T}\right]^{T}$. Consequently, when $\mathscr{W}_{1}$ is "non-increasing", $\mathscr{W}_{2}$ is also "non-increasing", and vise-versa. Therefore, it is guaranteed that the value of $\mathscr{W}_{1}\left(\mathscr{W}_{2}\right)$ at the beginning of each interval on which the nominal system (reconfigured system) is active does not exceed the value of $\mathscr{W}_{1}\left(\mathscr{W}_{2}\right)$ at the end of the previous such interval, if one exists.

Now let us introduce a new function,

$$
y_{\overline{\mathscr{E}}}(t)= \begin{cases}-\dot{\mathscr{W}}_{1}(t) & \text { if } t \in \overline{\mathscr{E}} \\ 0 & \text { otherwise }\end{cases}
$$

Given that $\mathscr{W}_{1}$ is always non-increasing, one obtains $\int_{0}^{t} y_{\overline{\mathscr{E}}}(\tau) d \tau \leq \mathscr{W}_{1}\left(t_{h_{1}}\right)-\mathscr{W}_{1}(t) \leq \mathscr{W}_{1}\left(t_{h_{1}}\right)$. Note that since $\dot{\mathscr{W}}_{1}(t) \leq 0, \forall t \geq 0$, we have $y_{\overline{\mathscr{E}}}(t)=\left|y_{\overline{\mathscr{E}}}(t)\right|, \forall t \geq 0$, and therefore, $y_{\overline{\mathscr{E}}}(t) \in \mathscr{L}_{1}$. Next, we show that $y_{\overline{\mathscr{E}}}(t) \rightarrow 0$ as $t \rightarrow \infty$.

Let us suppose that $y_{\overline{\mathscr{E}}}(t) \not \rightarrow 0$ as $t \rightarrow \infty$. Then, there exists a sequence $t_{n}$ in $\mathbb{R}_{+}$such that $t_{n} \rightarrow \infty$ as $n \rightarrow \infty$, and $\left|y_{\overline{\mathscr{E}}}\left(t_{n}\right)\right| \geq \varepsilon>0$ for all $n$, where $n \in \mathbb{N}$. It follows from the definition of $y_{\overline{\mathscr{E}}}(t)$ that the sequence $t_{n}$ necessarily belongs to $\overline{\mathscr{E}}$. Theorem 1 guarantees that $\delta q_{j}, \dot{q}_{j}, q_{j n}$, and $\dot{q}_{j n}$ remain bounded, therefore, $y_{\overline{\mathscr{E}}}(t)$ is uniformly continuous on $\overline{\mathscr{E}}$. By the uniform continuity of $y_{\overline{\mathscr{E}}}(t)$ on $\overline{\mathscr{E}}$, it follows that there exists a $\delta>0$ such that for all $n$ and all $0 \leq t \in \overline{\mathscr{E}}$, we have $\left|t_{n}-t\right| \leq \delta \Rightarrow\left|y_{\overline{\mathscr{E}}}\left(t_{n}\right)-y_{\overline{\mathscr{E}}}(t)\right| \leq \frac{\varepsilon}{2}$.

In other words, for all $t \in\left[t_{n}, t_{n}+\delta\right]$ and for all $n$ we have $\left|y_{\overline{\mathscr{E}}}(t)\right|=\left|y_{\overline{\mathscr{E}}}\left(t_{n}\right)-\left(y_{\overline{\mathscr{E}}}\left(t_{n}\right)-y_{\overline{\mathscr{E}}}(t)\right)\right| \geq\left|y_{\overline{\mathscr{E}}}\left(t_{n}\right)\right|-$ $\left|y_{\overline{\mathscr{E}}}\left(t_{n}\right)-y_{\overline{\mathscr{E}}}(t)\right| \geq \varepsilon-\frac{\varepsilon}{2}=\frac{\varepsilon}{2}$ (recall that the length of each interval in $\overline{\mathscr{E}}$ is bounded from below by $\bar{\tau}>0$ ). This 
contradicts the assertion stated earlier that $y_{\overline{\mathscr{E}}}(t) \in \mathscr{L}_{1}$. Therefore, $y_{\overline{\mathscr{E}}}(t) \rightarrow 0$ as $t \rightarrow \infty^{1}$.

Now by taking into account the strong connectivity of the communication graph, it follows from equations (3), (9) and (14) that $\dot{q}_{j} \rightarrow 0$ and $\dot{q}_{j n} \rightarrow 0, \forall j, n \in\{1, \ldots, m\}, j \neq n$ as $t \rightarrow \infty$. By the uniform continuity of $\dot{q}_{j}$ on $\overline{\mathscr{E}}$, we have $\ddot{q}_{j} \rightarrow 0, \forall j \in\{1, \ldots, m\}$ as $t \rightarrow \infty$. Therefore, the closed-loop dynamics of the $j$-th EL system can be written as $D_{j}\left(q_{j}\right) \ddot{q}_{j}=-\Lambda_{j}^{p} \chi\left(\delta q_{j}\right)-\sum_{n \in \mathscr{N}_{j}} \Lambda_{j n}^{p} \chi\left(q_{j n}\right) \rightarrow 0, \forall j, n \in\{1, \ldots, m\}, j \neq n$ as $t \rightarrow \infty$. Given that $\Lambda_{j n}^{p}=\Lambda_{n j}^{p}$ and $\chi\left(q_{j n}\right)=-\chi\left(q_{n j}\right)$, it is straightforward to show that $\sum_{j=1}^{m} \sum_{n \in \mathscr{N}_{j}} \Lambda_{j n}^{p} \chi\left(q_{j n}\right)=0$, which implies that $\sum_{j=1}^{l} \Lambda_{j}^{p} \chi\left(\delta q_{j}\right)=0$. Therefore, the requirements of Lemma 1 are satisfied, and one can conclude that $\delta q_{j} \rightarrow 0$, $q_{j n} \rightarrow 0, \forall j, n \in\{1, \ldots, m\}, j \neq n$, as $t \rightarrow \infty$. In other words, (a) all the states and control signals of the closedloop networked EL system will remain bounded, (b) the synchronization errors asymptotically converge to origin, i.e., $q_{j n} \rightarrow 0$ and $\dot{q}_{j n} \rightarrow 0$ as $t \rightarrow \infty$, and (c) the set-point tracking errors asymptotically converge to origin, i.e., $\delta q_{j} \rightarrow$ $0, \dot{q}_{j} \rightarrow 0$ as $t \rightarrow \infty$. This completes the proof of the theorem.

Remark 1. One can extend the results in Theorem 2 to develop a switching control law among a family (more than two and finite number) of saturation functions with guaranteed stability. Furthermore, the results in Theorem 2 can also be used to develop a switching control law among a family (a finite set) of monotonically increasing odd functions, e.g. $\chi_{\sigma}(x), \sigma=1,2, \ldots, \bar{m}$, with guaranteed stability.

\section{Simulation Studies}

The reconfigurable control scheme that we have developed in the previous sections is now applied to the problem of cooperative control of a team of robot manipulators, which represents a class of (electro-) mechanical EL systems. The nonlinear dynamical models corresponding to the robots are developed in the Matlab SimMechanics toolbox. We consider three non-identical (heterogenous) manipulators $(m=3)$ with two rotational joints. We further consider a fully bidirectionally connected communication graph with two leaders (the manipulators \#1 and \#2, i.e. $l=2$ ) and one follower (the manipulator \#3). It follows from our results presented in Section III that providing the desired coordinates vector to only one agent creates the possibility of a single point of failure in the network. Therefore, for the purpose of conducting simulations we consider a team having two leaders and one follower.

Through the use of our proposed control approach, the robots in the network synchronize their coordinate vectors while following the desired coordinate vector which is assumed to be identical for all the three manipulators, i.e. $q_{j}^{\star}=q^{\star}, j=1,2,3$. It is assumed that the torques that are applied to the joints are initially constrained during the normal operation of the actuators with $\bar{u}_{j}^{\max }=30 \mathrm{~N}-\mathrm{m}, j \in\{1,2,3\}$. However, due to an intermittent actuator fault in the manipulator $\# 1$, the maximum torque that is available to the first joint is reduced to $\left.\underline{u}_{1}^{\max }\right|_{1}=6 \mathrm{~N}-\mathrm{m}$. This fault is injected at the time $t_{\text {fault }}=370 \mathrm{sec}$ and is cleared at time $t=550 \mathrm{sec}$. The values of the nominal and the reconfigured controller gains are not given here due to space limitations. However, it worth noting that

\footnotetext{
${ }^{1}$ Note that the proof is similar to the proof of the well-known Barbalat's lemma [19], which cannot be used here since $y_{\overline{\mathscr{E}}}(t)$ is not a uniformly continuous function. A similar proof has appeared in Theorem 7 in [24].
} 
these gains are not the same. We use the linear saturation function, i.e., $\operatorname{Sat}_{1}(x)=\left\{\begin{array}{lc}x & \text { if }-1 \leq x \leq 1 \\ \operatorname{Sgn}(x) & \text { otherwise }\end{array}\right.$, for the nominal controller and $\operatorname{Sat}_{2}(x)=\frac{x}{\sqrt{\kappa^{2}+x^{2}}}$ for the reconfigured controller in our simulations with $\kappa=0.2$. The closed-loop responses of the manipulators under our proposed control strategies are depicted in Fig. 2(a). The associated control efforts of the manipulator \#1 for joints 1 and 2 are depicted in Fig. 2(b). It can be observed from Fig. 2(b) that from $t=370 \mathrm{sec}$ to $t=400 \mathrm{sec}$ the control efforts do not exceed the saturation limit of $6 \mathrm{~N}-\mathrm{m}$. It follows from Fig. 2(a) that prior to the injection of the fault, the angular positions settle down to their desired set-points by using the nominal controller.

However, at $t=400 \mathrm{sec}$ while the fault is still present the set-point of the joint 1 of all the manipulators is changed. Due to the coupling effects, the change in the set-point of joint 1 causes a change in the angular position of joint 2. The required torque to maintain the manipulator \#1 joint 1 at its desired angular position becomes higher than that of its actuator limit as seen from Fig. 2(b). Consequently, this leads to the actuator saturation and instability of the network of manipulators (from $t=400 \mathrm{sec}$ to $t=430 \mathrm{sec}$ ). It is now assumed that the control reconfiguration is implemented and invoked at $t_{\text {reconf. }}=430 \mathrm{sec}$, that is the controller is switched from the nominal to the reconfigured module.

Fig. 2(a) shows that after the controller reconfiguration at $t=430 \mathrm{sec}$, the closed-loop networked system is stabilized and the angular position errors converge to zero by utilizing the constrained control efforts of $6 \mathrm{~N}-\mathrm{m}$. Moreover, to further demonstrate the stability of our switched system, at time $t=550 \mathrm{sec}$ the fault is removed or cleared from the actuator of the manipulator \#1. Subsequently, following condition (a) of Assumption 2 (with $\bar{b}=0.4$ ) we switch from the reconfigured controller to the nominal controller at time $t=600 \mathrm{sec}$ (the top yellow box in the graph shows the duration when the fault is present and the bottom green box shows the duration when the reconfigured control is active). It can be observed from Fig. 2(a) that after $t=600 \mathrm{sec}$ the tracking errors converge to zero as required.

For providing a more descriptive explanation on the behavior of the cooperation error, in Fig. 3(a) the closed-loop EL system responses before and after the controller reconfiguration are provided. One can observe from Fig. 3(b) that the cooperation errors are smaller when the nominal controllers are used as compared to the reconfigured controllers. This is obviously due to the fact that the control effort constraints on the nominal system are satisfied, however, degradations in the performance of the faulty system are unavoidable due to the reduction of the control effort constraints. Therefore, it is highly recommended that one switches to the reconfigured controller only when a fault is present in the system, and when the fault is removed or cleared and during the healthy operation of the team the nominal controller is used exclusively.

\section{CONCLUSIONS}

A reconfiguration strategy for cooperative control of a network of nonlinear (electro-) mechanical Euler-Lagrange (EL) systems subject to actuator faults and constraints is developed in this work. The proposed nonlinear control strategy guarantees stability of the EL networked agents states and control signals and guarantees global convergence 
of the set-point tracking errors and the state synchronization errors to origin despite the presence of either actuator saturation constraints or intermittent and permanent actuator faults. By using our proposed switching strategy between the nominal and the reconfigured controllers, global stability of the closed-loop networked EL system states and control signals as well as convergence of the synchronization errors and the tracking errors to origin can still be ensured. Furthermore, the proposed control laws require minimum knowledge of the system's dynamics. The performance of our proposed reconfigurable control strategy is demonstrated by simulations to three heterogenous 2-DOF networked robots subject to actuator fault and actuator saturation.

\section{ACKNOWLEDGEMENT}

The authors gratefully acknowledge the anonymous reviewers' comments that have significantly improved the paper's readability.

\section{REFERENCES}

[1] A. Jadbabaie, J. Lin, and A. S. Morse, "Coordination of groups of mobile autonomous agents using nearest neighbor rules," IEEE Transactions on Automatic Control, vol. 48, no. 6, pp. 988-1001, 2003.

[2] W. Ren, "On consensus algorithms for double-integrator dynamics," IEEE Transactions on Automatic Control, vol. 53, no. 6, pp. 1503-1509, 2008.

[3] E. Semsar-Kazerooni and K. Khorasani, "Optimal consensus algorithms for cooperative team of agents subject to partial information," Automatica, vol. 44, no. 11, pp. 2766-2777, 2008.

[4] _ - "Switching control of a modified leaderfollower team of agents under the leader and network topological changes," IET Control Theory \& Applications, vol. 5, no. 12, pp. 1369-1377, 2011.

[5] D. Stipanovic, G. Inalhan, R. Teo, and C. Tomlin, "Decentralized overlapping control of a formation of unmanned aerial vehicles," Automatica, vol. 40, no. 8, pp. 1285-1296, 2004.

[6] J. Shan, "Six-degree-of-freedom synchronised adaptive learning control for spacecraft formation flying," IET Control Theory \& Applications, vol. 2, no. 10, pp. 930-949, 2008.

[7] A. R. Mehrabian, S. Tafazoli, and K. Khorasani, "Quaternion-based attitude synchronization and tracking for spacecraft formation subject to sensor and actuator constraints," AIAA Guidance, Navigation, and Control Conference, 2010.

[8] A. Rodriguez-Angeles and H. Nijmeijer, "Mutual synchronization of robots via estimated state feedback: A cooperative approach," IEEE Transactions on Control Systems Technology, vol. 12, no. 4, pp. 542-554, 2004.

[9] S.-J. Chung and J.-J. Slotine, "Cooperative robot control and concurrent synchronization of lagrangian systems," IEEE Transactions on Robotics, vol. 25, no. 3, pp. 1-15, 2009.

[10] W. Ren, "Distributed leaderless consensus algorithms for networked euler-lagrange systems," International Journal Control, vol. 82, no. 11, pp. 2137-2149, 2009.

[11] A. R. Mehrabian, S. Tafazoli, and K. Khorasani, "Reconfigurable control of networked nonlinear euler-lagrange systems subject to fault diagnostic imperfections," 50th IEEE Conference on Decision and Control and European Control Conference, 2011.

[12] — _ "Distributed $h_{i}$ nfty optimal control of networked uncertain nonlinear euler-lagrange systems with switching communication network topologies," 50th IEEE Conference on Decision and Control and European Control Conference, 2011.

[13] — " "State synchronization of networked euler-lagrange systems with switching communication topologies subject to actuator faults," 18th IFAC World Congress, 2011.

[14] N. Chopra, Output Synchronization of Networked Passive Systems. University of Illinois at Urbana-Champaign: PhD dissertation, 2006.

[15] Y. Zhang and J. Jiang, "Bibliographical review of reconfigurable fault-tolerant control systems," Annu. Rev. in Contr., vol. 32, no. 2, pp. 229-252, 2008.

[16] R. Ortega, P. J. Nicklasson, and H. Sira-Ramirez, Passivity-Based Control of Euler-Lagrange Systems: Mechanical, Electrical, and Electromechanical Applications. New York, NY: Springer-Verlag, 1998. 
[17] D. Scharf, F. Hadaegh, and S. Ploen, "A survey of spacecraft formation flying guidance and control (part ii): Control," Amer. Contr. conf., 2004.

[18] M. Huang, P. Caines, and R. Malhamé, "Large-population cost-coupled lqg problems with nonuniform agents: Individual-mass behavior and decentralized $\varepsilon$-nash equilibria," IEEE Transactions on Automatic Control, vol. 52, no. 9, pp. 1560-1571, 2007.

[19] S. Sastry, Nonlinear Systems: Analysis, Stability, and Control. New York, NY: Springer-Verlag, 1999.

[20] N. Meskin and K. Khorasani, "Actuator fault detection and isolation for a network of unmanned vehicles," IEEE Transactions on Automatic Control, vol. 54, no. 4, pp. 835-840, 2009.

[21] N. Meskin, K. Khorasani, and C. Rabbath, "Hybrid fault detection and isolation strategy for non-linear systems in the presence of large environmental disturbances," IET Control Theory and Applications, vol. 4, no. 12, pp. 2879-2895, 2010.

[22] _ - "A hybrid fault detection and isolation strategy for a network of unmanned vehicles in presence of large environmental disturbances," IEEE Transactions on Control Systems Technology, vol. 18, no. 6, pp. 1422-1429, 2011.

[23] A. Bacciotti and L. Mazzi, "An invariance principle for nonlinear switched systems," systems \& Control Letters, vol. 54, no. 11, pp. 1109-1119, 2005.

[24] J. Hespanha, D. Liberzon, D. Angeli, and E. Sontag, "Nonlinear norm-observability notions and stability of switched systems," IEEE Transactions on Automatic Control, vol. 50, no. 2, pp. 154-168, 2005. 

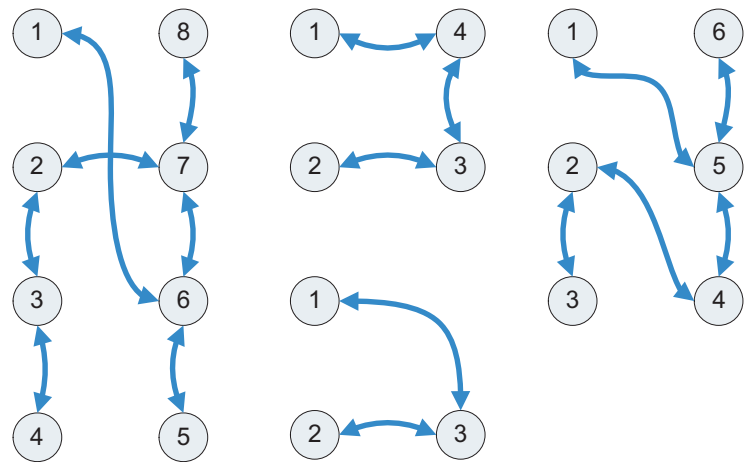

Fig. 1. Typical communication graphs corresponding to 3, 4, 6 and 8 agents in the network. 

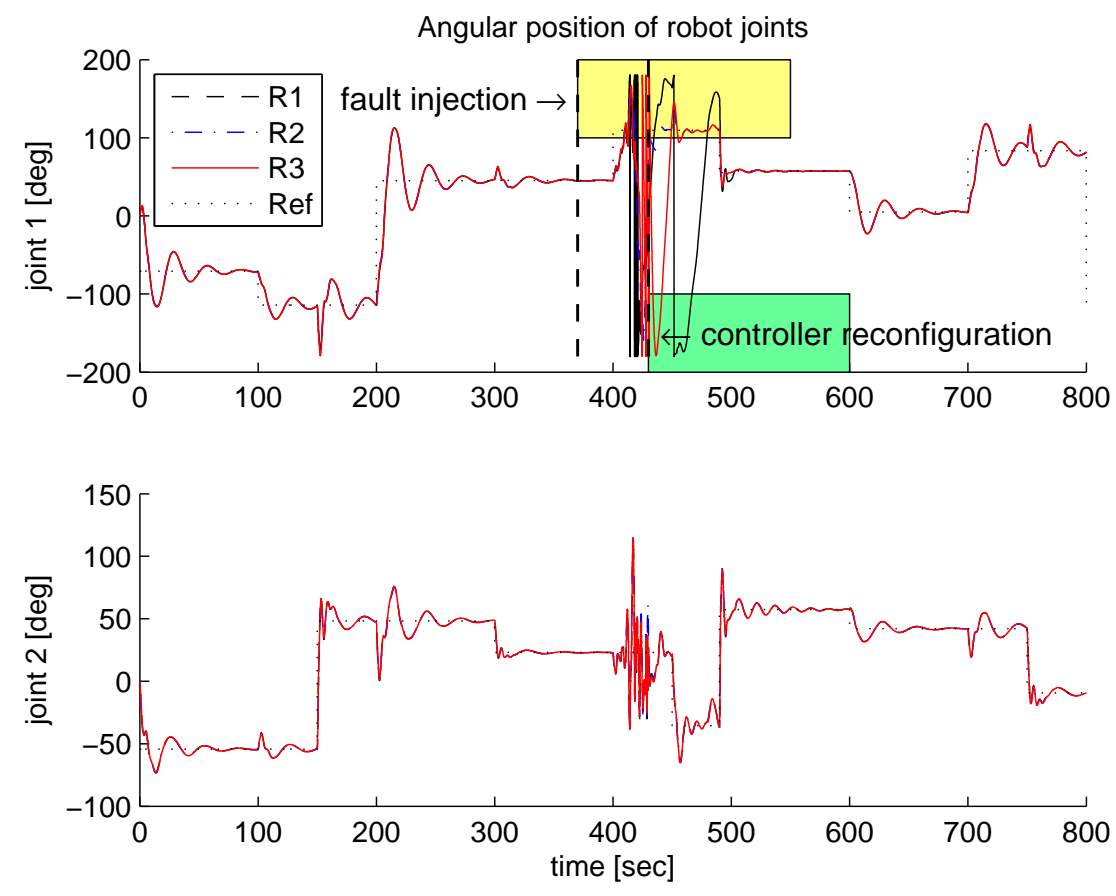

(a) Angular positions of the three robots.
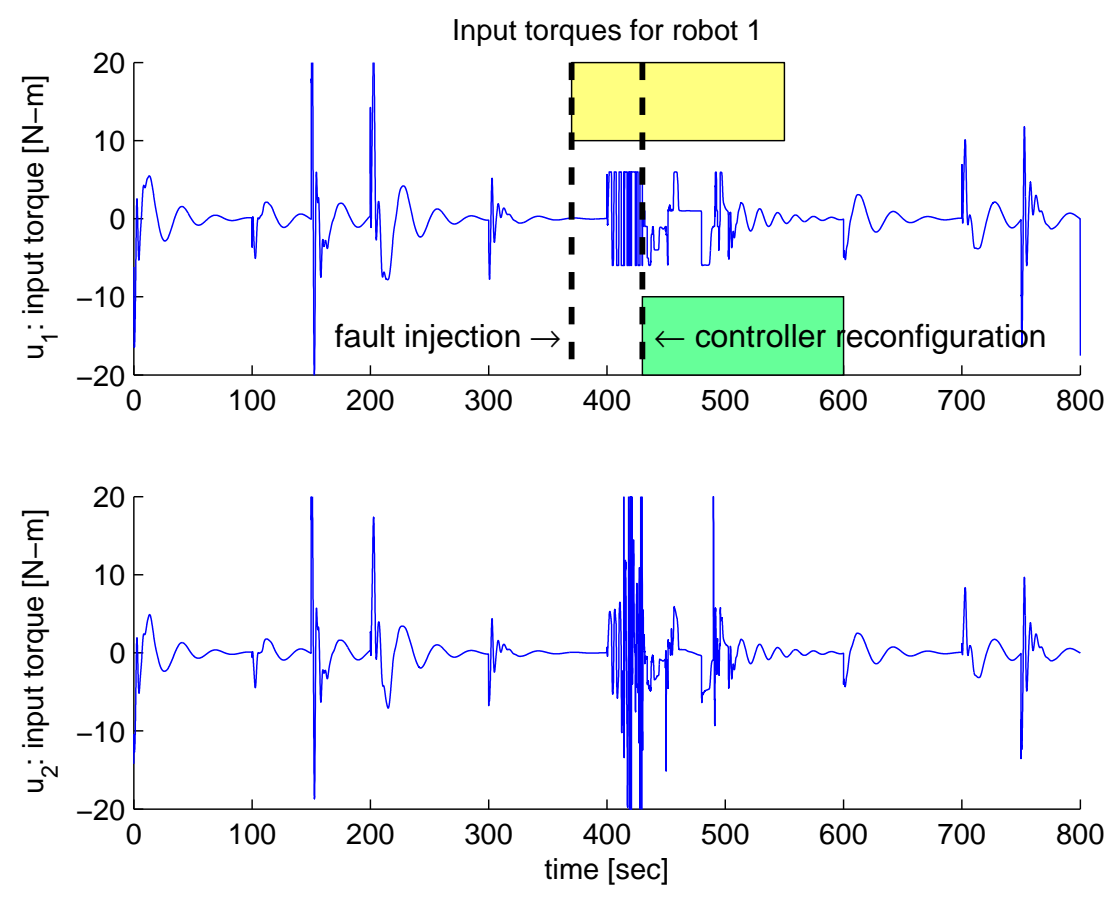

(b) Control effort of the robot \# 1 .

Fig. 2. Reconfigurable control of three robots (R1 to R3) when an intermittent fault is injected at time $t=370$ sec and cleared at time $t=550$ sec only in the actuator \# 1 of robot \# 1. 

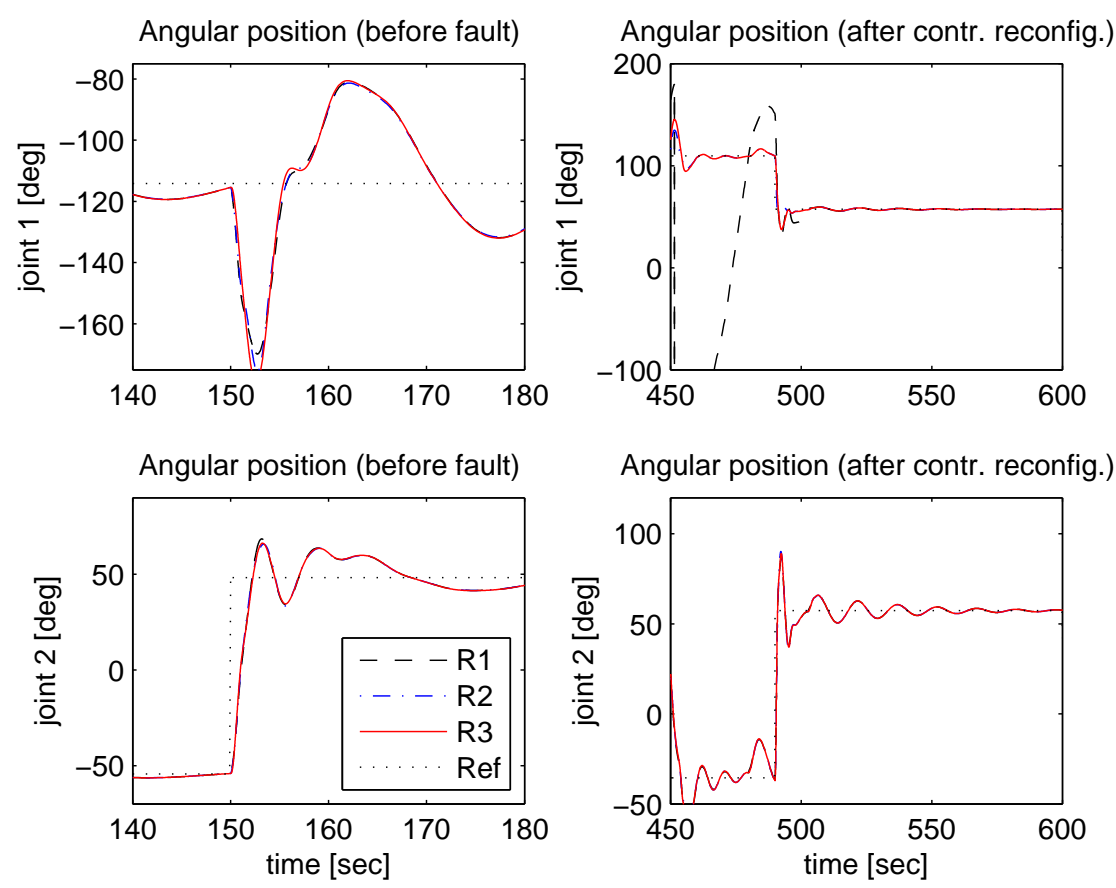

(a) Closed-loop angular position responses.
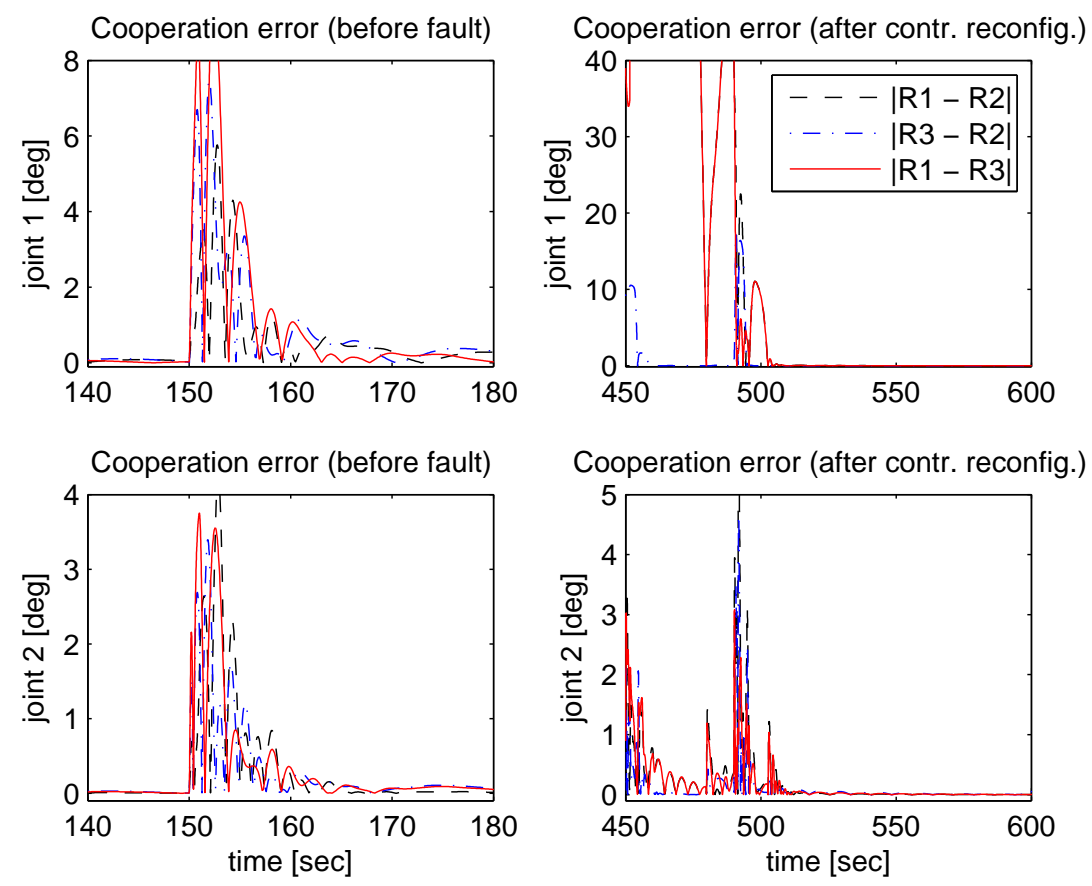

(b) Cooperation errors in the angular positions.

Fig. 3. Scaled responses before an intermittent fault is injected only in the joint \# 1 of robot \# 1 and after the control reconfiguration. 